[15] D. Bhandari, C. A. Murthy, and S. K. Pal, "Genetic algorithm with elitist model and its convergence," Int. J. Pattern Recognit. Artif. Intell., vol. 10, pp. 731-747, 1996.

[16] S. Bandyopadhyay, C. A. Murthy, and S. K. Pal, "Pattern classification with genetic algorithms," Pattern Recognit. Lett., vol. 16, pp. 801-808, 1995.

[17] C. A. Murthy and N. Chowdhury, "In search of optimal clusters using genetic algorithm," Pattern Recognit. Lett., vol. 17, pp. 825-832, 1996.

[18] S. Daly, "The visual difference predictor: An algorithm for the assessment of image fidelity," in SPIE Conf. Human Vision, Visual Processing and Digital Display III, San Jose, CA, 1992, pp. 2-15.

[19] C. A. Murthy and S. K. Pal, "Histogram thresholding by minimizing gray level fuzzyness," Inform. Sci., vol. 60, pp. 107-135, 1992.

[20] X. Ran and N. Farvardin, "A perceptually motivated three-componen image model-Part I: Description of the model," IEEE Trans. Image Processing, vol. 4, pp. 401-415, 1995.

[21] L. Thomas and F. Deravi, "Region-based fractal image compression using heuristic search," IEEE Trans. Image Processing, vol. 4, pp. 832-838, 1995 .

\section{An Error Resilient Scheme for Image Transmission over Noisy Channels with Memory}

Philippe Burlina and Fady Alajaji

\begin{abstract}
This correspondence addresses the use of a joint sourcechannel coding strategy for enhancing the error resilience of images transmitted over a binary channel with additive Markov noise. In this scheme, inherent or residual (after source coding) image redundancy is exploited at the receiver via a maximum a posteriori (MAP) channel detector. This detector, which is optimal in terms of minimizing the probability of error, also exploits the larger capacity of the channel with memory as opposed to the interleaved (memoryless) channel. We first consider MAP channel decoding of uncompressed two-tone and bitplane encoded grey-level images. Next, we propose a scheme relying on unequal error protection and MAP detection for transmitting grey-level images compressed using discrete cosine transform (DCT), zonal coding, and quantization. Experimental results demonstrate that for various overall (source and channel) operational rates, significant performance improvements can be achieved over interleaved systems that do not incorporate image redundancy.
\end{abstract}

Index Terms - Channels with memory, DCT coding, error resilience, joint source/channel coding, MAP decoding, unequal error protection.

\section{INTRODUCTION}

We address the problem of the reliable communication of images over bursty channels. Traditional approaches to the design of visual communication systems over noisy channels rely on Shannon's

Manuscript received January 24, 1996; revised May 16, 1997. This work was supported in part by the Natural Sciences and Engineering Research Council (NSERC) of Canada. Parts of this work were presented at the 1995 International Symposium on Information Theory and the 1996 International Conference on Image Processing. The associate editor coordinating the review of this manuscript and approving it for publication was Dr. Christine Podilchuk

P. Burlina is with the Institute for Advanced Computer Studies and the Electrical Engineering Department, University of Maryland, College Park MD 20742 USA (e-mail: burlina@cfar.umd.edu).

F. Alajaji is with the Department of Mathematics and Statistics and the Department of Electrical and Computer Engineering, Queen's University, Kingston, Ont. K7L 3N6, Canada.

Publisher Item Identifier S 1057-7149(98)02465-8. source-channel coding separation principle [9], resulting in what is known as tandem source-channel coding schemes. The optimality of this design principle holds only asymptotically; i.e., when no constraints exist on coding/decoding complexity and delay [9]. An alternate approach lies in joint source-channel coding (JSSC): this strategy includes techniques such as maximum a posteriori (MAP) detection, channel optimized vector quantization, or adaptive sourcechannel rate allocation. JSSC has recently received increased attention (e.g., [5], [7], [11]), and has been shown to outperform tandem schemes when delay and complexity are constrained. Most of the work on joint source-channel coding of images [5], [7], [11] has dealt with memoryless channels, disregarding the fact that real-world communication channels-in particular, mobile radio or satellite channels-often have memory.

In this work, we investigate the problem of MAP detection of images transmitted over a binary Markov channel. The MAP detector fully exploits the statistical image characteristics in order to efficiently combat channel noise. It also exploits the larger capacity of the channel with memory as opposed to the interleaved (memoryless) channel. We first describe MAP detection schemes that directly utilize the inherent image redundancy in uncompressed binary images and bit-plane encoded grey-level images. The amount of needed overhead information and the performance degradation when the decoder has imperfect knowledge of the channel parameters are considered.

The MAP detection approach is then validated for systems employing image compression. The residual redundancy of quantized lowfrequency discrete cosine transform (DCT) coefficients is exploited via unequal error protection (UEP) and MAP decoding. Experimental results show that the proposed schemes exhibit very good performance, in spite of their low complexity (which primarily resides in the MAP decoder). Specifically, significant gains over systems not exploiting image redundancy can be achieved, at relatively low overall transmission rates.

\section{Channel Model}

Consider a binary channel with memory described by $Y_{i}=X_{i} \oplus Z_{i}$, for $i=1,2, \cdots$ where $X_{i}, Z_{i}$ and $Y_{i}$ represent, respectively, the input, noise and output of the channel. The input and noise sequences are assumed to be independent from each other. The noise process $\left\{Z_{i}\right\}$ is a stationary ergodic Markov process described in [2], with channel bit error rate (BER) denoted by $\epsilon$, where $\epsilon \in[0,1 / 2)$, and correlation parameter denoted by $\delta \geq 0$ (the noise correlation coefficient is given by $\frac{\delta}{1+\delta}$ ). When $\delta=0$, the channel reduces to the memoryless binary symmetric channel (BSC). The channel transition and marginal probabilities $Q\left(z_{n} \mid z_{n-1}\right) \triangleq \operatorname{Pr}\left\{Z_{n}=z_{n} \mid Z_{n-1}=\right.$ $\left.z_{n-1}\right\}$ and $Q\left(z_{n}\right) \triangleq \operatorname{Pr}\left\{Z_{n}=z_{n}\right\}$, are given by

$$
\left[\begin{array}{ll}
Q(0 \mid 0) & Q(1 \mid 0) \\
Q(0 \mid 1) & Q(1 \mid 1)
\end{array}\right]=\frac{1}{1+\delta}\left[\begin{array}{cc}
1-\epsilon+\delta & \epsilon \\
1-\epsilon & \epsilon+\delta
\end{array}\right]
$$

and $Q(1)=\epsilon=1-Q(0)$. Note that this Markov model is general; it can represent any irreducible first-order two-state Markov chain. The channel capacity is given [2] by

$$
C=1-H\left(Z_{2} \mid Z_{1}\right)=1-(1-\epsilon) h_{b}\left(\frac{\epsilon}{1+\delta}\right)-\epsilon h_{b}\left(\frac{1-\epsilon}{1+\delta}\right)
$$

where $h_{b}(\cdot)$ is the binary entropy function. The capacity is monotonically increasing with $\delta$ (for fixed $\epsilon$ ) and monotonically decreasing with $\epsilon$ (for fixed $\delta$ ). Note that for fixed $\epsilon$, as $\delta \rightarrow \infty, C \rightarrow 1$. 


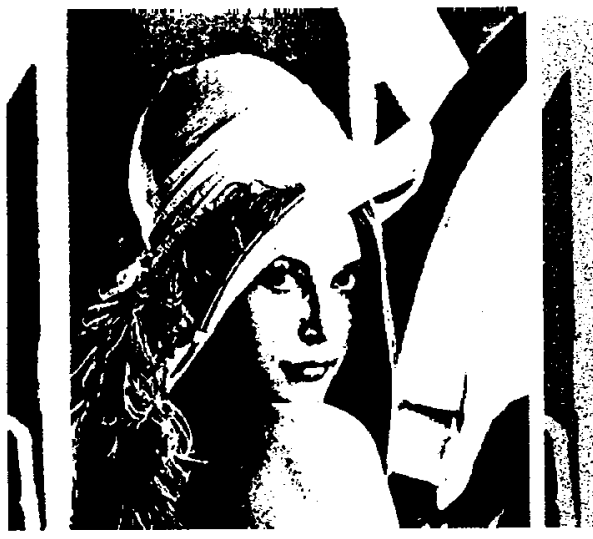

(a)

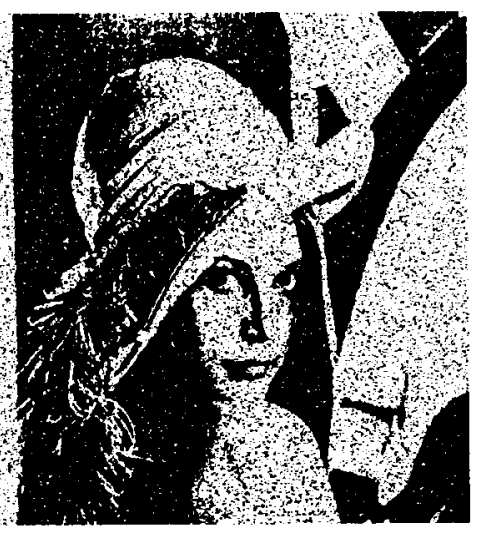

(b)

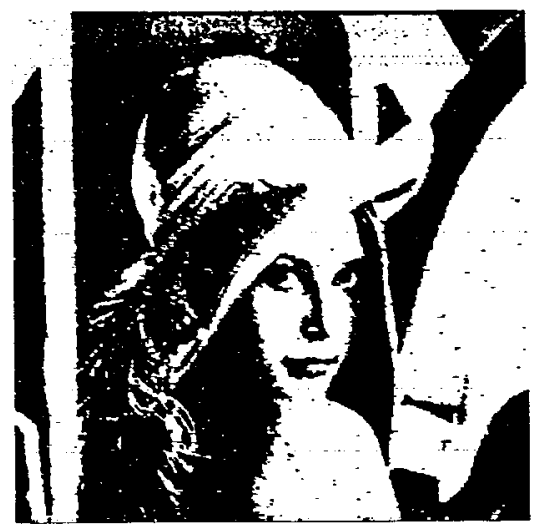

(c)

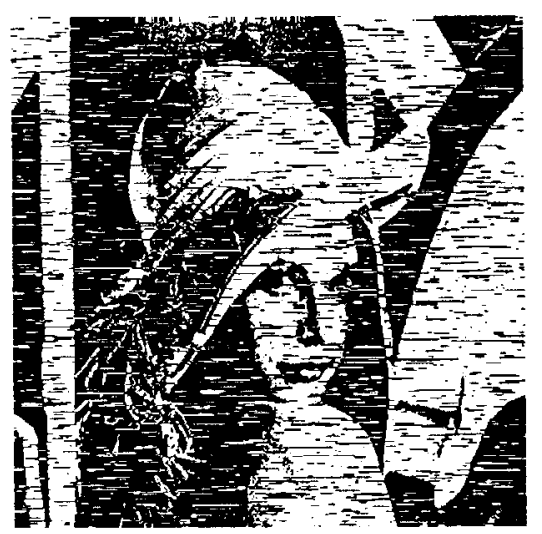

(d)

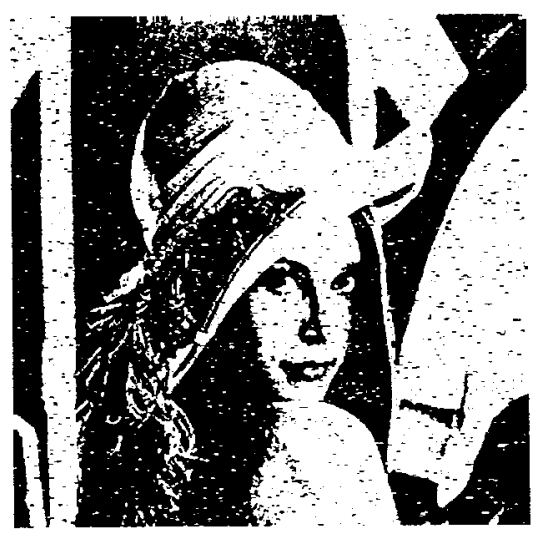

(e)

Fig. 1. MAP detection of two-tone Lena over the Markov channel with $\epsilon=0.1$. (a) Binary Lena. (b) Lena received, $\delta=0$. (c) MAP detected using second-order model. (d) Received uncoded Lena, $\delta=10$. (e) Decoded Lena: adaptive scheme.

\section{UNCOMPRESSED IMAGES}

\section{A. Image Models, MAP Detection, and Image Redundancy}

Consider a two-tone image $U=\left[U_{i, j}\right]$ of height $J$ and width $K$, where $U_{i, j}=0$ or $1, i=1, \cdots, J, j=1, \cdots, K$. We assume that the image satisfies a causal second-order Markov property such that any pixel at location $(i, j)$ depends on the pixels at locations $(i-1, j)$ and $(i, j-1)$. When the image is explored lexicographically, it can be represented as a second-order Markov process ${ }^{1}\left\{X_{n}\right\}$ where

$$
\begin{aligned}
& \operatorname{Pr}\left\{X_{n}=x_{n} \mid X_{n-1}=x_{n-1}, \cdots, X_{1}=x_{1}\right\} \\
& \quad=\operatorname{Pr}\left\{X_{n}=x_{n} \mid X_{n-1}=x_{n-1}, X_{n-K}=x_{n-K}\right\}
\end{aligned}
$$

for $n>K$. Note that this model is completely specified by four transitional distributions. We also consider the following special cases of $\left\{X_{n}\right\}$ : the first-order Markov chain and nonuniform independent and identically distributed (i.i.d.) models [4].

Consider the problem of transmitting the binary second-order Markov source $\left\{X_{n}\right\}$ over the Markov channel. The optimal detection technique that minimizes the sequence probability of decoding error is the sequence MAP method [3]. More specifically, if $Y^{n}=$ $y^{n}=\left(y_{1}, y_{2}, \cdots, y_{n}\right)$ denotes the received binary sequence at the output of the channel, the MAP detector "guesses" the transmitted

\footnotetext{
${ }^{1}$ General Markov random field (MRF) models [8] are not used here, since MAP estimation for these models would require computationally intensive algorithms such as simulated annealing. We therefore restrict ourselves to causal models that are easily implemented via sequential decoding algorithms.
}

sequence $\hat{x}^{n}$ according to

$$
\hat{x}^{n}=\arg \max _{x^{n} \in\{0,1\}^{n}} \operatorname{Pr}\left\{X^{n}=x^{n} \mid Y^{n}=y^{n}\right\} .
$$

It can be shown [4] that (1) is equivalent to

$$
\begin{aligned}
\hat{x}^{n}= & \arg \max _{x^{n} \in\{0,1\}^{n}}\left[\log \left(Q\left(x_{1} \oplus y_{1}\right) P\left(x_{1}\right)\right)\right. \\
& +\sum_{k=2}^{K} \log \left(Q\left(y_{k} \oplus x_{k} \mid y_{k-1} \oplus x_{k-1}\right) P\left(x_{k} \mid x_{k-1}\right)\right) \\
& +\sum_{k=K+1}^{n} \log \left(Q\left(y_{k} \oplus x_{k} \mid y_{k-1} \oplus x_{k-1}\right)\right. \\
& \left.\left.\times P\left(x_{k} \mid x_{x-1}, x_{k-K}\right)\right)\right] .
\end{aligned}
$$

The sequence MAP detector described in (2) can be implemented using the Viterbi algorithm. Here, $x_{k}$ denotes the state at time $k$; the trellis will hence have two states, with two branches leaving and entering each state. For a branch leaving state $x_{k-1}$ at time $k-1$ and entering state $x_{k}$ at time $k$, the path metric is

$$
-\log \left(Q\left(y_{k} \oplus x_{k} \mid y_{k-1} \oplus x_{k-1}\right) P\left(x_{k} \mid x_{x-1}\right)\right), \text { for } k \leq K,
$$
and

$$
-\log \left(Q\left(y_{k} \oplus x_{k} \mid y_{k-1} \oplus x_{k-1}\right) P\left(x_{k} \mid x_{x-1}, x_{k-K}\right)\right),
$$

for $k>K$.

The surviving path for each state is the path with the smallest cumulative metric up to that state. The sequence MAP decoder observes 
the entire received sequence $y^{n}$ in order to estimate $x_{1}, x_{2}, \cdots, x_{n}$.

In this scheme, channel protection is achieved by utilizing the natural source redundancy as well as the channel noise correlation. The total redundancy contained in the source $\left\{X_{n}\right\}$ is $\rho_{T}=$ $1-H_{\infty}(X)$, where $H_{\infty}(X)$ is the source entropy rate. This total redundancy can be written as $\rho_{T}=\rho_{D}+\rho_{M}$ [3], where $\rho_{D} \triangleq$ $1-H\left(X_{1}\right)$ denotes the redundancy due to the nonuniformity of the marginal distribution $\left(\operatorname{Pr}\left\{X_{1}=x\right\}\right)$, and $\rho_{M} \triangleq H\left(X_{1}\right)-H_{\infty}(X)$, denotes the redundancy due to the memory of the process. The type and amount of redundancy exhibited by an image is important since it dictates the behavior of the MAP detector. If $\rho_{M} \gg \rho_{D}$, the process tends to behave like a symmetric Markov source. This results in a mismatch situation (cf. [3, Section V]) that prevents the decoder from fully exploiting the channel noise correlation (when the channel capacity increases, the performance of the MAP detector deteriorates.) If the redundancy due to the nonuniformity of a process is high relative to its redundancy in the form of memory $\left(\rho_{D} \gg \rho_{M}\right)$, then the process behaves like a nonuniform i.i.d. source and no such mismatch occurs [3, Sec. IV]. Images and facsimile documents exhibit very different types and degrees of redundancy. ${ }^{2}$ Furthermore, redundancy varies within images themselves since images are hardly stationary sources. This observation suggests the use of an adaptive scheme, as will be proposed next.

\section{B. Two-Tone Image Detection}

We start by modeling the two-tone images according to the secondorder causal Markov chain. Image lines are each represented as a Markov chain with transitional probabilities computed empirically, and transmitted uncompressed in a lexicographic fashion over the Markov channel. At the receiver, the sequence MAP decoder is implemented according to (2). While the 2-D Markov model is appealing, since it closely captures the spatial dependency specific to image sources, simulation results suggest that the use of this model often results in a mismatch between the source and the channel [4]. This leads us to conclude that when images are modeled by a second-order Markov chain and sent over the binary Markov channel, the best performance is obtained when $\delta=0$; i.e., when the channel is fully interleaved and transformed into a memoryless channel (BSC). Fig. 1(a)-(c) show the binary Lena image transmitted over the interleaved channel with BER $\epsilon=0.1$. The resulting average decoding bit error probability is 0.039 .

We next consider MAP decoding when the image is modeled as a first-order Markov chain. Since images are nonstationary, image lines can be classified in two ways: (a) Lines for which neither $\rho_{M}$ nor $\rho_{D}$ are dominant, in which case no mismatch occurs. (b) Lines having $\rho_{M} \gg \rho_{D}$, which are likely to result in mismatch. We hence employ an adaptive encoding system on the image lines that takes into consideration the line redundancy. Each image line, modeled as a first-order Markov chain, is processed as follows: The empirical distributions for the line are computed. If $\rho_{M}<T * \rho_{D}$, for some threshold $T$, we transmit the image line over the channel and MAP decode it using the line statistics and first-order Markov assumptions. Otherwise, if $\rho_{M} \geq T * \rho_{D}$, we first convert the redundancy in the symmetric Markov source $\left\{X_{n}\right\}$ from the form of memory into redundancy in the form of nonuniform distribution via the transformation, as follows [4]: ${ }^{3} V_{n}=X_{n} \oplus X_{n-1}, n=1,2, \cdots, K$. We then transmit $V^{K}$ directly over the Markov channel, and MAP decode it as $\hat{V}^{K}$ using i.i.d. source assumptions. The decoded binary image stream is reconstructed using $\hat{X}_{k}=\hat{V}_{k} \oplus \hat{X}_{k-1}$,

\footnotetext{
${ }^{2}$ Computational studies that quantify natural redundancy inherent in twotone images are reported in [4]

${ }^{3}$ This is essentially equivalent to differential encoding for binary sources.
}

TABLE I

Percentage of Overhead for Binary Lena. $K=512 ; R=2$.

\begin{tabular}{c|c|c|c}
\hline$l$ & $m=4$ & $m=2$ & $m=1$ \\
\hline \hline 1 & $6.25 \%$ & $3.13 \%$ & $1.57 \%$ \\
\hline 2 & $10.94 \%$ & $5.47 \%$ & $2.74 \%$ \\
\hline
\end{tabular}

TABLE II

Binary Lena: Robustness Results for AdaPtive MAP Decoding Scheme IN PSNR (dB); $T=10 ; \epsilon_{d}=$ DESIGN BER; $\epsilon_{a}=$ ActuAl BER; $\delta_{d}=$ Design Correlation Parameter; $\delta_{a}=$ Actual Correlation Parameter. (a) Robustness With BER $\left(\delta_{d}=\delta_{a}=10\right)$.

(b) Robustness with Correlation Parameter $\left(\epsilon_{d}=\epsilon_{a}=0.1\right)$

\begin{tabular}{c||c|c|c|c}
\cline { 2 - 5 } \multicolumn{1}{c||}{} & $\epsilon_{d}=0.00$ & $\epsilon_{d}=0.01$ & $\epsilon_{d}=0.05$ & $\epsilon_{d}=0.10$ \\
\hline \hline$\epsilon_{a}=0.00$ & $\infty$ & 27.71 & 21.89 & 19.33 \\
\hline$\epsilon_{a}=0.01$ & 22.12 & 23.09 & 20.41 & 18.54 \\
\hline$\epsilon_{a}=0.05$ & 14.94 & 17.35 & 17.45 & 16.27 \\
\hline$\epsilon_{a}=0.10$ & 12.07 & 14.73 & 14.68 & 14.79 \\
\hline
\end{tabular}

(a)

\begin{tabular}{l|c|c|c|c}
\cline { 2 - 5 } \multicolumn{1}{c||}{} & $\delta_{d}=0$ & $\delta_{d}=2$ & $\delta_{d}=5$ & $\delta_{d}=10$ \\
\hline$\delta_{a}=0$ & 8.25 & 6.71 & 6.67 & 6.67 \\
\hline$\delta_{a}=2$ & 8.49 & 10.69 & 10.65 & 10.61 \\
\hline$\delta_{a}=5$ & 8.46 & 12.91 & 12.92 & 12.92 \\
\hline$\delta_{a}=10$ & 8.55 & 14.57 & 14.58 & 14.79 \\
\hline
\end{tabular}

(b)

$k=1,2, \cdots, K$ with $\hat{X}_{1}=\hat{V}_{1}$. To prevent error propagation, packetization is used by grouping source samples into blocks. An example of adaptive MAP decoding $(T=10)$ of Lena over a very noisy channel with high noise correlation $(\epsilon=0.1$ and $\delta=10)$ is shown in Fig. 1(d) (received as if it were not protected) and (e) (MAP decoded). A $4.68 \mathrm{~dB}$ peak signal-to-noise ratio (PSNR) gain is achieved by the adaptive MAP decoder over the case when no MAP decoding is done. Detailed performance evaluation of this scheme for various images is reported in [4].

\section{Overhead Information}

As in all joint source-channel coding schemes, it is assumed that the image statistics are available at the decoder. This can be achieved by transmitting them along with the image using a forward errorcorrecting code. ${ }^{4}$ We assume that a rate $1 / R$ convolutional encoder is used to protect the source statistics. If the channel is very noisy, we might need to use a more powerful convolutional code. This can be achieved by increasing the number of states of the code or increasing $R$. If $l$ denotes the number of accuracy digits for each source parameter, then the percentage of overhead information is equal to

$$
\% \text { Overhead }=\frac{m R\left\lceil\log _{2}\left(10^{l}-1\right)\right\rceil}{K}
$$

where $K$ is the image width and $m$ is the number of source statistics per line ( $m=4$ for the second-order Markov model, $m=2$ for the first-order model, and $m=1$ for iid model). The amount of overhead needed for the Lena two-tone image is presented in Table I for $R=2$ and $l=1,2$.

${ }^{4}$ Note that we can avoid transmitting overhead information about the source statistics by using training images to estimate the statistics of the source. This approach is justifiable in applications where the images belong to a particular class-e.g., in the transmission of medical magnetic resonance images (MRI's). 


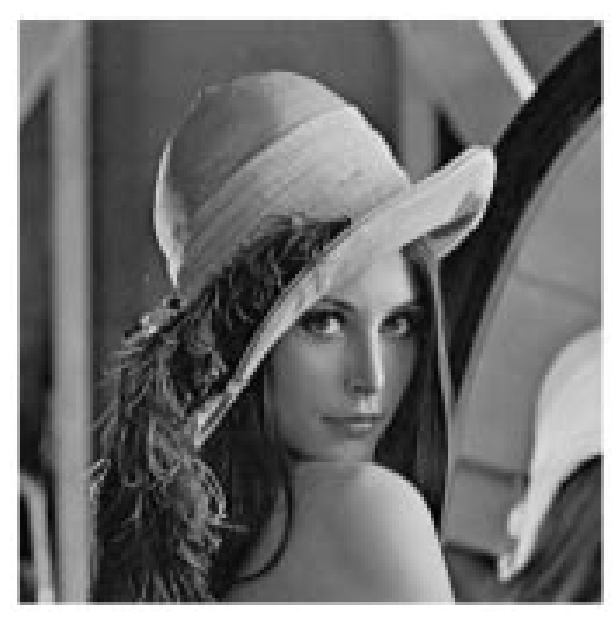

(a)

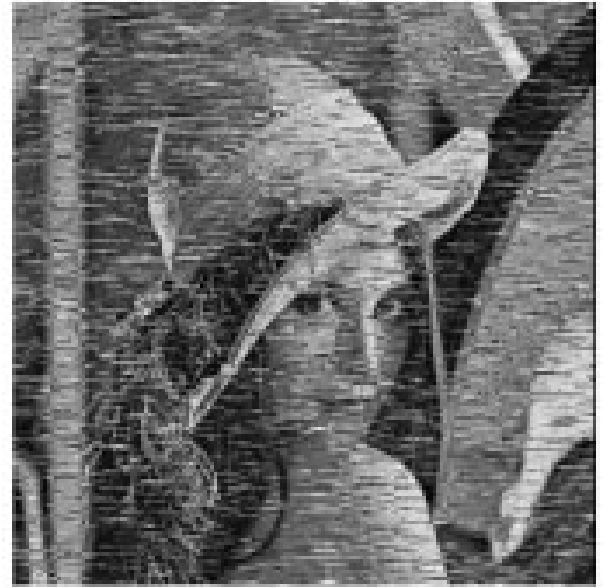

(b)

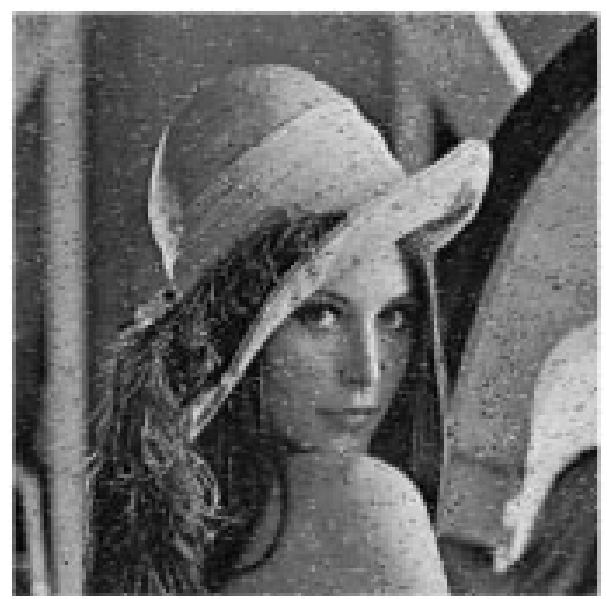

(c)

Fig. 2. Transmission of grey Lena using MAP detection of bit-plane encoded images; $\epsilon=0.1, \delta=10$. (a) Original Lena. (b) Received uncoded; PSNR $=14.45 \mathrm{~dB}$. (c) Decoded Lena; PSNR $=19.53 \mathrm{~dB}$.

\section{Robustness Under Imperfectly Known Channel Statistics}

Until now we have assumed that the channel statistics $(\epsilon$ and $\delta)$ were known a priori at the receiver. We investigate here the robustness of the MAP decoding system when these parameters are not known perfectly. This may occur due to inadequate estimation of the channel parameters, particularly when the channel is time-varying (e.g., mobile radio channels). Simulation results using the adaptive MAP decoding scheme for the transmission of Lena are displayed in Table II. In Table II(a), we present PSNR results when the receiver misestimates the BER $\epsilon$ with the correlation parameter $\delta=10$. In Table II(b), we provide PSNR results when the receiver misestimates the correlation parameter $\delta$ with the channel BER $\epsilon=0.1$. We can conclude that the MAP scheme is not very sensitive to errors in estimating $\epsilon$ or $\delta$, provided that we do not design $\epsilon$ or $\delta$ to be zero when the actual parameter is nonzero.

\section{E. Bit-Plane Encoded Grey-Level Images}

For illustrative purposes, we herein consider the application of the MAP decoding method to bit-plane encoded images. In bit-plane coding, each plane is traditionally compressed using binary image coding techniques [6]. This method is very sensitive to channel errors and typically yields low compression ratios leaving little room for
TABLE III

MAP-UNC Versus UNC: Average PSNR (IN dB) of DeCoded Lena Over Markov Channel with BER $\epsilon$ and Correlation Parameter $\delta$. Results Averaged over 30 Experiments. $R$ Is the Overall Rate In B/Pixel

\begin{tabular}{|c|c|c|c|c|c|c|c|}
\hline$R$ (bpp) & System & $\delta$ & $E=0$ & $c=0.005$ & $c=0.01$ & $c=0.05$ & $c=0.1$ \\
\hline \multirow[t]{6}{*}{0.35} & UNC & 0 & 26.16 & 23.07 & 21.29 & 15.75 & 12.91 \\
\hline & MAP-UNC & 0 & 26.16 & 23.90 & 22.45 & 17.57 & 15.28 \\
\hline & UNC & 5 & 26.16 & 23.12 & 21.55 & 15.82 & 13.06 \\
\hline & MAP-UNC & 5 & 26.16 & 23.51 & 22.09 & 18.00 & 16.20 \\
\hline & UNC & 10 & 26.16 & 23.53 & 21.65 & 15.81 & 12.87 \\
\hline & $\mathrm{M} A \mathrm{P}-\mathrm{UNC}$ & 10 & 26.16 & 23.91 & 22.38 & 18.55 & 16.93 \\
\hline \multirow[t]{6}{*}{0.63} & $\mathrm{UNC}$ & 0 & 28.52 & 23.92 & 21.83 & 15.58 & 12.78 \\
\hline & MAP-UNC & 0 & 28.52 & 25.06 & 23.12 & 17.74 & 15.59 \\
\hline & UNC & $\overline{0}$ & 28.52 & 23.98 & 21.73 & 15.64 & 12.91 \\
\hline & MAP-UNC & 5 & 28.52 & 24.49 & 22.73 & 18.33 & 16.24 \\
\hline & UNC & 10 & 28.52 & 24.10 & 21.73 & 15.71 & 12.80 \\
\hline & MAP-UNC & 10 & 28.52 & 24.96 & 23.04 & 18.98 & 17.00 \\
\hline \multirow[t]{6}{*}{1.19} & UNC & 0 & 31.75 & 24.66 & 22.20 & 15.63 & 12.73 \\
\hline & MAP-UNC & 0 & 31.75 & 26.29 & 23.93 & 17.76 & 15.25 \\
\hline & ENC & 5 & 31.75 & 25.02 & 22.34 & 15.42 & 12.82 \\
\hline & MAP-UNC & 5 & 31.75 & 25.59 & 23.28 & 18.38 & 16.38 \\
\hline & UNC & 10 & 31.75 & 24.66 & 22.14 & 15.52 & 12.79 \\
\hline & MAP-INC & 10 & 31.75 & 26.20 & 24.07 & 19.04 & 17.17 \\
\hline
\end{tabular}


TABLE IV

MAP-UEP I VERSUS ML-IL-UEP I: AvERAGE PSNR (IN dB) OF DECODED Lena over Markov Channel with BER $\epsilon$ AND Correlation Parameter $\delta$. Results Averaged over 30 Experiments. $R$ Is the Overall Rate In B/Pixel

\begin{tabular}{|c|c|c|c|c|c|c|c|}
\hline$R$ (bpp) & System & $\delta$ & $t=0$ & $t=0.005$ & $t=0.01$ & $t=0.05$ & $c=0.1$ \\
\hline \multirow[t]{6}{*}{0.47} & ML-UL-UEP I & 0 & 26.16 & 25.36 & 24.70 & 19.91 & 14.86 \\
\hline & MAP-UEP I & 0 & 26.16 & 25.73 & 25.30 & 22.64 & 18.38 \\
\hline & ML-IL-UEP I & 5 & 26.16 & 25.36 & 24.70 & 19.91 & 14.86 \\
\hline & MAP-UEP I & 5 & 26.16 & 25.98 & 25.72 & 23.22 & 18.33 \\
\hline & ML-IL-UEP I & 10 & 26.16 & 25.36 & 24.70 & 19.91 & 14.86 \\
\hline & $M A P-U E P$ I & 10 & 26.16 & 26.00 & 25.79 & 23.37 & 18.59 \\
\hline \multirow[t]{6}{*}{0.75} & MI_-IL-UEP I & 0 & 28.52 & 26.82 & 25.59 & 19.66 & 14.36 \\
\hline & MAP-UEP I & 0 & 28.52 & 27.59 & 26.82 & 23.11 & 18.40 \\
\hline & ML-IL-UEP I & 5 & 28.52 & 26.82 & 25.59 & 19.66 & 14.36 \\
\hline & MAP-UEP I & 5 & 28.52 & 28.11 & 27.69 & 24.02 & 18.67 \\
\hline & ML-IL-UEP I & 10 & 28.52 & 26.82 & 25.59 & 19.66 & 14.36 \\
\hline & MAP-UEP I & 10 & 28.52 & 28.22 & 27.86 & 24.04 & 18.85 \\
\hline \multirow[t]{6}{*}{1.31} & ML-IL-UTEP I & 0 & 31.75 & 28.47 & 26.51 & 19.48 & 14.40 \\
\hline & $M A P^{3}-U E P I$ & 0 & 31.75 & 29.70 & 28.37 & 23.17 & 18.28 \\
\hline & ML-IL-UEP I & 5 & 31.75 & 28.47 & 26.51 & 19.48 & 14.40 \\
\hline & MAP-UEP I & 5 & 31.75 & 30.77 & 29.93 & 24.49 & 18.83 \\
\hline & ML-IL-UEP I & 10 & 31.75 & 28.47 & 26.51 & 19.48 & 14.40 \\
\hline & MAP-UEP I & 10 & 31.75 & 31.02 & 30.32 & 24.77 & 18.95 \\
\hline
\end{tabular}

protection against channel noise. Consider instead the problem of directly sending the uncompressed bit-planes modeled as Markov sources over the Markov channel. As in the case of two-tone images, we use an adaptive MAP detection scheme taking into account the source and the channel statistics applied on each bit-plane image explored in a lexicographic fashion. Experimental results are shown in Fig. 2 for the Lena grey-level image. Significant improvements over the received images are achieved. For $\delta=10$ and $\epsilon=0.1$, gains in excess of $5 \mathrm{~dB}$ are achieved.

\section{COMPRESSED IMAges}

MAP decoding of uncompressed images relies on the significant intrinsic source redundancy to help combat channel noise. Since source coding schemes are not ideal, they always leave some residual redundancy in their output bitstream that can similarly be exploited at the receiver. A challenging issue lies in the use of the limited redundancy residing in compressed images for channel protection.

\section{A. Image Compression Scheme}

Standard visual compression methods such as Joint Photographers Expert Group (JPEG) and Motion Pictures Expert Group (MPEG) are fragile to channel errors. Errors corrupting the compressed data contribute unequally to the final distortion of the reconstructed image or video stream. This observation justifies the use of unequal error protection. We propose to improve the error resilience of compressed images by designing several schemes that combine UEP and MAP detection. Our objective is to characterize the effectiveness of these methods for various levels of image compression.

Consider the case of JPEG encoded images, or that of MPEG1/2 or H.261/3 encoding of intraframes. These schemes incorporate DCT coding, quantization and entropy coding. Clearly, the most fragile module lies in the variable-length coding (either Huffman or arithmetic), for which the occurrence of an error produces catastrophic error propagation and total loss of the packet until the next
TABLE V

MAP-UEP II vERSUS ML-IL-UEP II: AVERAGE PSNR (IN dB) OF DECODED Lena over Markov Channel with BER $\epsilon$ AND Correlation Parameter $\delta$. Results Averaged over 30 Experiments. $R$ Is the Overall Rate in B/PIXel

\begin{tabular}{|c|c|c|c|c|c|c|c|}
\hline$R(\mathrm{bpp})$ & System & $\delta$ & $\epsilon=0$ & $\epsilon=0.005$ & $c=0.01$ & $c=0.05$ & $\epsilon=0.1$ \\
\hline \multirow[t]{6}{*}{0.69} & ML-IL-UEP II & 0 & 26.16 & 26.13 & 26.06 & 22.33 & 15.40 \\
\hline & MAP-UEP II & 0 & 26.16 & 26.15 & 26.08 & 23.88 & 18.81 \\
\hline & MI $\mathrm{IL}_{-} \mathrm{UJEP}$ II & 5 & 26.16 & 26.13 & 26.06 & 22.33 & 15.40 \\
\hline & MAP-UEP II & 5 & 26.16 & 26.14 & 26.06 & 24.00 & 18.72 \\
\hline & ML-IL-UEP II & 10 & 26.16 & 26.13 & 26.06 & 22.33 & 15.40 \\
\hline & MAP-UEP II & 10 & 26.16 & 26.14 & 26.09 & 23.90 & 18.64 \\
\hline \multirow[t]{6}{*}{0.97} & ML-IL-UEP II & 0 & 28.52 & 27.89 & 27.30 & 21.60 & 14.98 \\
\hline & MAP-UEP II & 0 & 28.52 & 28.26 & 27.90 & 24.36 & 18.83 \\
\hline & ML-II-UEP II & 5 & 28.52 & 27.89 & 27.30 & 21.60 & 14.98 \\
\hline & MAP-UEP II & 5 & 28.52 & 28.39 & 28.20 & 24.66 & 18.80 \\
\hline & ML-IL-UEP II & 10 & 28.52 & 27.89 & 27.30 & 21.60 & 14.98 \\
\hline & MAP-UEP II & 10 & 28.52 & 28.39 & 28.23 & 24.67 & 18.84 \\
\hline \multirow[t]{6}{*}{1.5 .3} & MI_-II_-UFP II & 0 & 31.75 & 30.16 & 28.87 & 21.52 & 14.89 \\
\hline & MAP-UEP II & 0 & 31.75 & 30.80 & 30.09 & 24.88 & 18.83 \\
\hline & ML-IL-UEP II & 5 & 31.75 & 30.16 & 28.87 & 21.52 & 14.89 \\
\hline & MAP-UEP II & 5 & 31.75 & 31.24 & 30.78 & 25.40 & 18.94 \\
\hline & ML-IL-UEP II & 10 & 31.75 & 30.16 & 28.87 & 21.52 & 14.89 \\
\hline & MAP-UEP II & 10 & 31.75 & 31.36 & 30.96 & 25.66 & 19.06 \\
\hline
\end{tabular}

synchronization occurs. Error resilience in this case consist in the reliable reception of synchronization messages or the packetization of VL codes. ${ }^{5}$

Since the synchronization issue is outside the scope of this work, we consider instead a compression scheme similar in spirit to the above cited standards with the exclusion of entropy coding. More specifically, our image compression scheme is as follows: The image is first subdivided into $8 \times 8$ blocks, and for each of these blocks the DCT is computed. The resulting 64 DCT coefficients are uniformly quantized using one of the quantization matrices proposed in [10] derived from psychovisual thresholds. The coefficients are then ordered in a zig-zag fashion. While the basic JPEG scheme would Huffman encode the resulting stream on the basis of the coefficients' amplitude and leading run-lengths of zeros, we proceed with zonal coding and conversion to a binary bitstream. For zonal coding, we use the first 15 zig-zag scanned coefficients. The retained quantized coefficients are then converted to binary using a folded binary code (FBC) representation. The bit rates used for converting each quantized coefficient are those proposed for zonal coding in [10].

\section{B. Channel Coding Schemes}

Error resilience is provided by combining UEP and MAP detection. Because of the high-energy compaction property of the DCT for highly correlated sources [6], most of the signal information is concentrated in the lower spatial frequencies. The DC coefficient is the most important DCT coefficient since it measures the average value of each block. An error in the DC coefficient typically results in blocking artifacts. These artifacts are often resolved through additional channel protection or postprocessing techniques that employ edge-preserving smoothing operators on the decoded image. However, traditional channel protection or error-concealment operations disregard the source characteristics. We propose instead to use MAP detection of channel encoded DC

\footnotetext{
${ }^{5}$ This issue is given much attention in current standardization efforts of
} MPEG4 


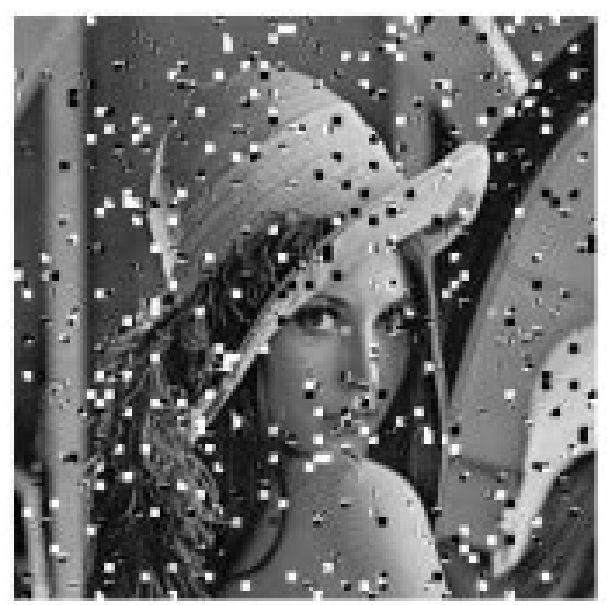

(a)

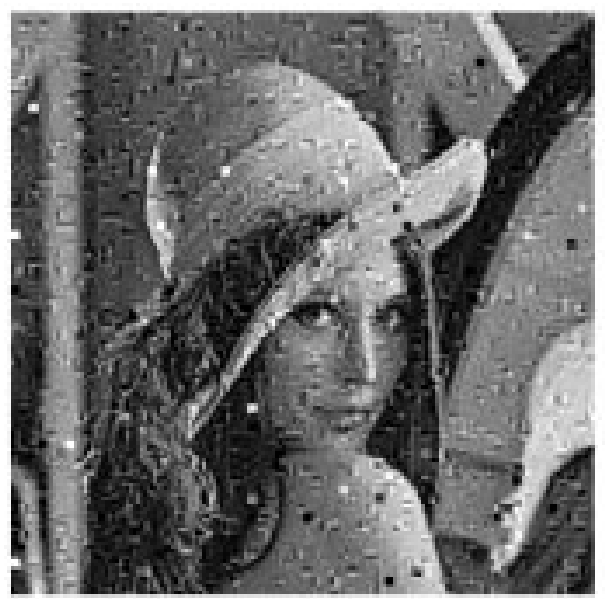

(c)

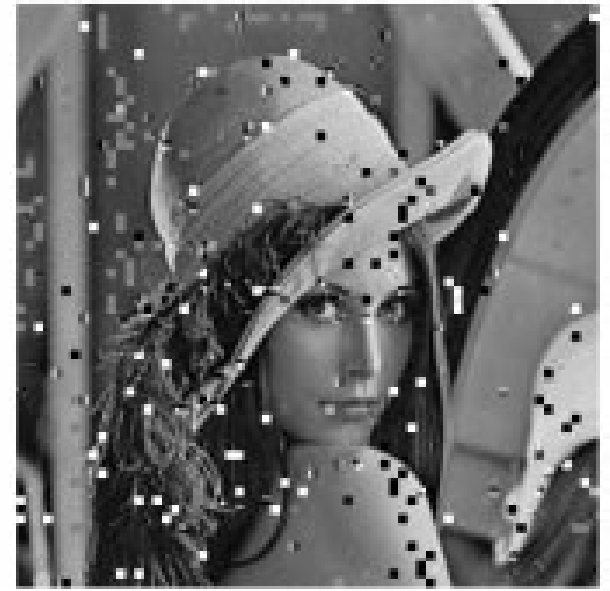

(b)

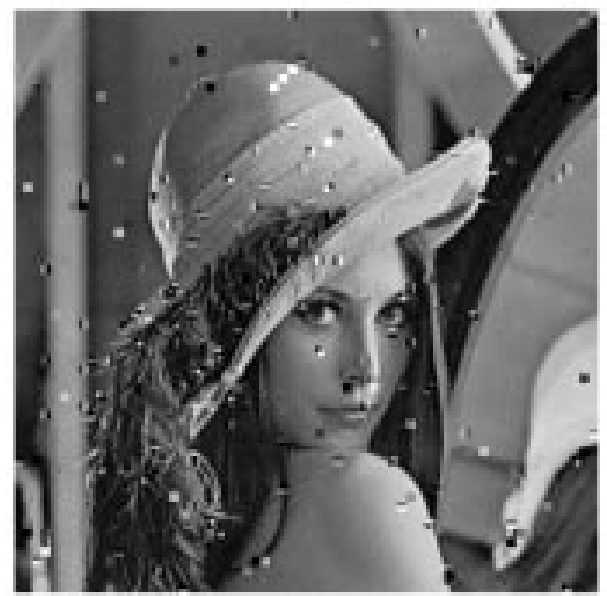

(d)

Fig. 3. Transmission of grey Lena using $S_{1}$ ordering method over Markov channel with $\epsilon=0.05 ; \delta=20$. (a) UNC; PSNR $=15.30 \mathrm{~dB}, R=1.19 \mathrm{~b} /$ pixel. (b) MAP-UNC; PSNR $=17.75 \mathrm{~dB}, R=1.19 \mathrm{~b} /$ pixel. (c) ML-UEP-IL; PSNR $=19.72 \mathrm{~dB}, R=1.31 \mathrm{~b} /$ pixel. (d) MAP-UEP; PSNR $=22.90 \mathrm{~dB}, R=1.31 \mathrm{~b} /$ pixel.

coefficients. For enhanced protection, additional low frequency DCT coefficients are included in this UEP scheme. This choice results in slightly higher complexity and increased overall (source + channel) coding rates. The following schemes are considered.

1) MAP-UNC: In this case, no channel coding is performed. For each block, the FBC bitstream is modeled as an i.i.d. nonuniform source, sent over the Markov channel and decoded via a MAP detector. The channel memory and the residual redundancy due to the nonuniform distribution of the FBC data are utilized by the MAP detector to combat channel noise. The performance of this scheme is compared to that obtained using an uncoded system of equal overall rate, and referred to as $U N C$.

2) MAP-UEP-I: Here, we use UEP by providing additional protection to all image DC coefficients: they are channel encoded via low complexity four-state, rate- $1 / 2$ convolutional encoder. The convolutionally coded DC bitstream is modeled as an iid source, sent over the interleaved Markov channel, and MAP decoded. This is achieved by interleaving the DC bitstream among the data of the AC coefficients. Interleaving is performed for the DC coefficients since the convolutional code introduces memory in the bitstream; hence, a bursty channel noise behavior can cause an error propagation in the decoder.
As for the AC coefficients, they are sent without interleaving over the Markov channel and MAP detected.

3) MAP-UEP-II: In this scheme, the first $N(N>1)$ low order zig-zag scanned DCT coefficients are selected for UEP. UEP is provided as in MAP-UEP-I by using MAP channel detection of the convolutionally encoded coefficients.

The two latter schemes, MAP-UEP-I and MAP-UEP-II are compared to systems with identical overall rate and similar complexity denoted by ML-UEP-IL-I and -II: These schemes represent traditional tandem source-channel coding schemes with the same UEP technique as MAP-UEP-I and MAP-UEP-II but using maximum likelihood (ML) decoding over the interleaved Markov channel (with $\delta=0$ ).

We use two different binary bitstream ordering methods for transmitting the FBC data over the binary channel. In the first method (denoted by $S_{1}$ ), the bitstream is simply formed by sequentially sending the data block by block: Each block is scanned lexicographically, and each DCT coefficient within that block is converted to its FBC representation. The second stream method (denoted by $S_{2}$ ) is designed to exploit the interblock redundancy. While DCT coefficients should be uncorrelated, ${ }^{6}$ coefficients across neighboring blocks do exhibit significant dependency (and hence redundancy).

\footnotetext{
${ }^{6}$ They are uncorrelated for ideal sources generated by first order Markov processes.
} 


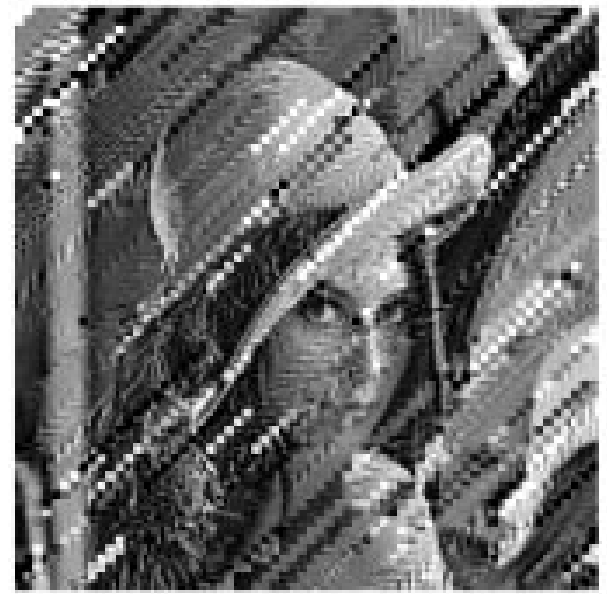

(a)

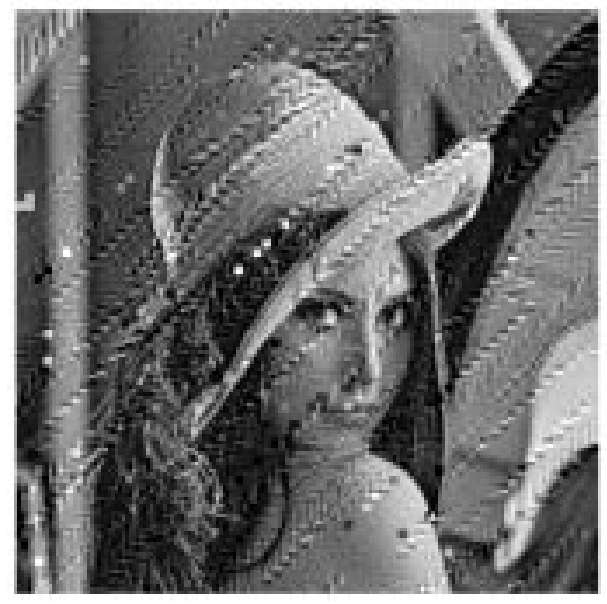

(c)

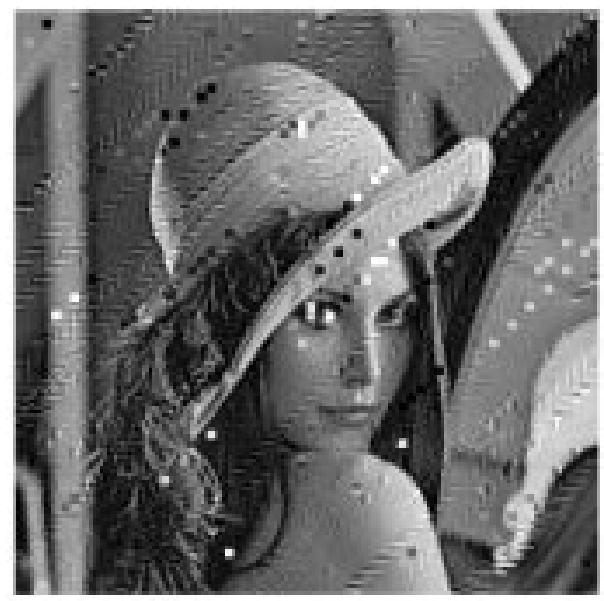

(e)

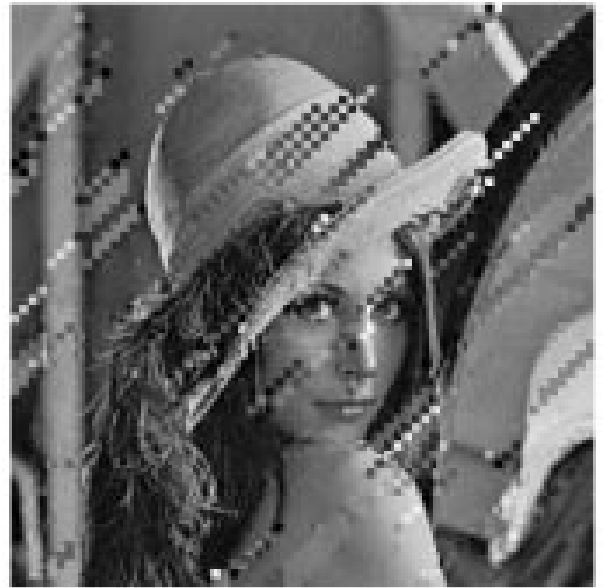

(b)

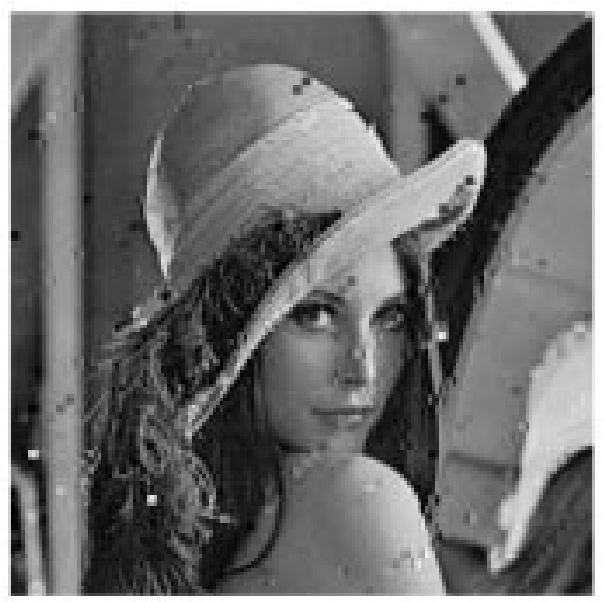

(d)

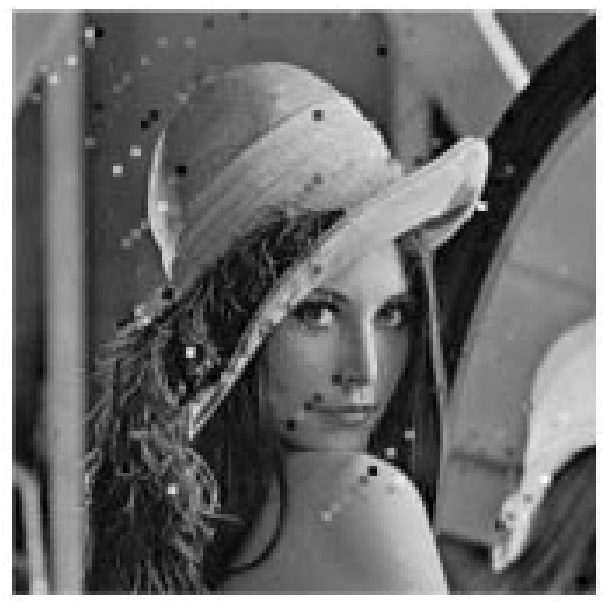

(f)

Fig. 4. Transmission of grey Lena using $S_{2}$ ordering method over Markov channel with $\epsilon=0.05$ and $\delta=10$. (a) UNC; PSNR $=15.54 \mathrm{~dB}, R=1.19$ b/pixel. (b) MAP-UNC; PSNR $=19.01 \mathrm{~dB}, R=1.19 \mathrm{~b} /$ pixel. (c) ML-IL-UEP I; PSNR $=19.47 \mathrm{~dB}, R=1.31 \mathrm{~b} /$ pixel. (d) MAP-UEP I; PSNR $=24.76 \mathrm{~dB}$, $R=1.31 \mathrm{~b} /$ pixel. (e) ML-IL-UEP II; PSNR $=21.55 \mathrm{~dB}, R=1.53 \mathrm{~b} /$ pixel. (f) MAP-UEP II; PSNR $=25.68 \mathrm{~dB}, R=1.53 \mathrm{~b} /$ pixel.

This is traditionally exploited in JPEG by using differential pulse code modulation (DPCM) coding of the DC coefficients across blocks. This redundancy is herein exploited in the $S_{2}$ method: the bitstream is obtained by taking the bitstream $S_{1}$ and grouping the bits corresponding to the same DCT coefficient and the same bit position in the coefficient's FBC representation, and by zig-zag scanning the blocks in the image. Note that this representation is amenable to progressive and scalable decoding of the image whereby the DCT coefficients for the full image are transmitted and decoded in order of increasing spatial frequency.

The value of the source compression ratio and the amount of image residual redundancy directly constrain the performance of 
the MAP detector. Source coding rate control should be carried out by modifying the original quantization matrix and accordingly determining the optimal bit rate allocation for each coefficient. We use instead a suboptimal scheme to achieve higher compression rates: Varying rates are obtained by modifying the size of the zonal mask and discarding additional high-frequency DCT coefficients. If 15 coefficients are kept, and the bit allocation set to $(8,7,7,6,6,6,4,5,5,4,3,4,4,4,3)$, a total of $76 \mathrm{~b}$ are used per block, yielding a rate of $1.19 \mathrm{~b} /$ pixel. When only six (respectively, three) coefficients are kept, the source coding rate decreases to 0.62 (respectively, 0.34) b/pixel.

The average PSNR obtained for the transmission of Lena using schemes 1,2 , and 3 is reported in Tables III, IV, and V. Note that in each case, schemes of comparable rates and complexity are compared to each other ${ }^{7}$. We look at the performance for various values of the channel correlation $\delta, \operatorname{BER} \epsilon$, and overall operational rate $R$ (in b/pixel). In each table, three different overall rates are used: they correspond to three different source coding rate allocations, representing zonal masks of size respectively equal to 15,6 , and 3 . These tables are obtained by using the bit ordering method $S_{2}$. Lastly, for MAP-UEP-II, $N=3$ is selected. In general, we can clearly observe from the results that the MAP- ${ }^{*}$ schemes offer superior performance, particularly when the channel is quite noisy (high $\epsilon$ ) and strongly correlated (high $\delta$ ). The best overall performance is obtained by the MAP-UEP schemes. Significant performance improvements are obtained by introducing even limited UEP, especially at low $\mathrm{BER}$, at the cost of often only moderate increases in overall rate (compare MAP-UNC at $(\delta, \epsilon, R)=(*, 0.01,1.19)$ to MAP-UEP-I at $(\delta, \epsilon, R)=(*, 0.01,1.31))$. It may be observed from these tables that MAP methods are able to exploit higher channel correlation. This is less pronounced in cases when UEP is used. Since MAP methods almost consistently yield a performance superior to that obtained by their ML counterpart for situations of interleaved channels $(\delta=$ 0 ), clearly the use of prior distribution translates into appreciable performance gain. It is apparent also from Table III that for *-UNC schemes, lower source coding rates translate in lower improvement margins between the MAP method and the UNC method. However, even at $0.35 \mathrm{~b} /$ pixel overall transmission rate, for $\delta=0$, the MAP method is still able to provide appreciable (over $2 \mathrm{~dB}$ ) PSNR coding gain over the UNC method. The same coding gain is observed for schemes using UEP.

Similar subjective performance improvements can be observed in Figs. 3 and 4. The bit ordering $S_{1}$ was used in Fig. 3, while $S_{2}$ was used in Fig. 4. Blocking artifacts resulting from the loss of low DCT coefficients are easily canceled using UEP. Also notable is the fact that while $S_{2}$ bit ordering provides more redundancy (due to the interblock DCT correlation) to be exploited by the receiver, any burst of error arising on one of the DC coefficient MSB's results in a diagonal streak in the image. This is not the case for images using bit ordering $S_{1}$. But again, these streaks are easily canceled using UEP.

\section{CONCLUSION}

We investigate the problem of reliably transmitting images over binary bursty channels. Several schemes exploiting the redundancy of uncompressed and compressed image sources are employed. Experimental results indicate that substantial objective as well as subjective performance improvements can be achieved over uncoded systems and systems that employ interleaving and do not take into consideration the image characteristics. In particular, we demonstrate

\footnotetext{
${ }^{7}$ The channel rate $r_{\mathrm{UNC}}^{c}$ resulting from the received uncoded scheme UNC is equal to the channel rate $r_{\text {MAP-UNC }}^{c}$ for the MAP-UNC scheme. Similarly, $r_{\text {MAP_UEP-I }}^{c}=r_{\text {ML-UEP-IL-I and }}^{c} r_{\text {MAP_UEP-II }}^{c}=r_{\text {ML-UEP-IL-II }}^{c}$. The source coding rates are also identical.
}

that low-complexity error resilient systems that use limited channel protection resources and no entropy coding can be designed if the inherent redundancy of the (uncompressed or compressed) image is exploited at the decoder, and this can be achieved even at moderate overall transmission rates. The compressed system utilized here is generic and incorporates some of the features of standard image coding schemes such as JPEG. It is expected that similar results would extend for the case of subband-coded images.

Future work will address the use of soft decision information in conjunction with trellis coded modulation (TCM) for the MAP channel decoding of compressed images over noisy channels. ${ }^{8}$

\section{ACKNOWLEDGMENT}

Prof. R. Chellappa is gratefully acknowledged for his insightful guidance and for many helpful discussions.

\section{REFERENCES}

[1] F. Alajaji, S. Al-Semari, and P. Burlina, "An unequal error protection trellis coding scheme for still image communication," in Proc. Int. Symp. Inform. Theory, Ulm, Germany, 1997.

[2] F. Alajaji and T. Fuja, "A communication channel modeled on contagion," IEEE Trans. Inform. Theory, vol. 40, pp. 2035-2041, Nov. 1994.

[3] F. Alajaji, N. Phamdo, N. Farvardin, and T. Fuja, "Detection of binary Markov sources over channels with additive Markov noise," IEEE Trans. Inform. Theory, vol. 42, pp. 230-239, 1996.

[4] P. Burlina, F. Alajaji, and R. Chellappa, "Transmission of two-tone images over noisy communication channels with memory," Tech. Rep. CAR-TR-814, Ctr. Automation Res., Univ. Maryland, College Park, 1996.

[5] S. Emmani and S. L. Miller, "DPCM picture transmission over noisy channels with the aide of a Markov model," IEEE Trans. Image Processing, vol. 4, pp. 1473-1481, 1995.

[6] A. K. Jain, Fundamentals of Digital Image Processing. Englewood Cliffs, NJ: Prentice-Hall, 1989.

[7] J. Modestino, D. Daut, and A. Vickers, "Combined source-channel coding of images using the block cosine transform," IEEE Trans. Commun., vol. 29, pp. 1261-1274, 1981.

[8] A. K. Jain and R. Chellappa, Eds., Theory and Applications of Markov Random Fields. New York: Academic, 1993.

[9] C. E. Shannon, "A mathematical theory of communication," Bell Syst. Tech. J., vol. 27, pp. 379-423 and pp. 623-656, 1948.

[10] A. M. Tekalp, Digital Video Processing. Englewood Cliffs, NJ: Prentice-Hall, 1995.

[11] W. Xu, J. Hagenauer, and J. Hollmann, "Joint source-channel decoding using the residual redundancy in compressed images," in Proc. Int. Conf. Communications, Dallas, TX, 1996.

${ }^{8}$ We have extended the ideas presented in this paper to the transmission of images over the AWGN channel using TCM systems in conjunction with MAP soft decision decoding [1]. More specifically, we propose in [1] new UEP TCM schemes that rely on selective power allocation and employ a soft-decision MAP decoding metric. These schemes result in considerable performance gains over uncoded systems and systems that use equal error protection. 